\title{
Emergence of the Laws of Nature in the Developing Entangled Universe
}

\author{
Evgeny A. Novikov \\ University of California - San Diego, BioCircuits Institute, La Jolla, CA \\ enovikov@ucsd.edu
}

\begin{abstract}
Evolution of our universe with continuous production of matter by the vacuum, is described. The analysis is based on the quantum modification of the general relativity (Qmoger), supported by the cosmic data without fitting. Various types of matter are selected by the vacuum in accordance with stability of the developing universe. All laws of nature seem to be emergent and approximate, including the conservation of energy. The (3+1)-dimensional space-time and gravity were selected first. After that came quantum condensate of entangled gravitons (dark matter). Photons and other ordinary matter were selected much later during formation of galaxies, when the background condensate becomes gravitationally unstable. The effect of radiation on the global dynamics is described in terms of conservation of the enthalpy density. Mass of neutrino, as the first massive fermionic particle created from the background condensate, is estimated, in accord with experimental bound. The electric dipole moment of neutrino is also estimated. The oscillations of neutrinos are explained in terms of interaction with background condensate. The phenomena of quantum entanglement of ordinary matter are, apparently, inherited from the background condensate. The phenomena of subjective experiences are also explained in terms of interaction of the action potentials of neurons with the background dipolar condensate, which opens a new window into the dark sector of matter. The Qmoger theory goes beyond the Standard Model and the Quantum Field Theory and can be combined with their achievements.
\end{abstract}

Keywords: quantum modification of general relativity, emergence of the laws of nature, isenthalpic universe, quantum condensate of gravitons, oscillating neutrinos, subjective experiences and dark sector of matter.

\section{INTRODUCTION}

The level of a civilization, basically, is determined by its cosmology. If we, the people, want to survive and prosper, we need to adapt a right cosmology. The fundamental question of physics, including cosmology, is where the laws of nature came from? In this paper we will try to answer this question. Our analysis is based on the quantum modification of general relativity (Qmoger), which is supported by cosmic observations without fitting (including the acceleration of the universe, see below). At the same time, we will advance the theory and get new results.

The Qmoger equations differ from the Einstein equations by two additional terms, which are responsible for production and absorption of matter (see section 3). This is in contrast with the conventional cosmology, which assumes that all matter of the universe was produced 13.7 billion years ago in a singular Big Bang (BB) [1]. The BB hypothesis inspired many important observations, including the resent detection of cosmic gravitational signals. But the BB theory becomes more and more cumbersome in its attempts to overcome contradictions with cosmic data [1-5]. This is a sure sign of a flawed theory. Particularly disturbing are, of course, the singularity of mysterious BB, the physically unexplainable critical density of the universe, the dark energy (ridiculously small cosmological constant with unclear physical sense) and the miraculous inflation (a rescue mission, which does not sit well with BB). The situation with BB theory reminds analogous situation with the geocentric model of Ptolemy. Old habits die hard and a resistance to the new cosmology is expected. But, in my humble opinion, it is time to move on.

\section{SPACE-TIME AND GRAVITY}

It seems natural to connect the creation of the universe with some kind of instability of the vacuum. That instability is inducing a release of something, which we, the people, now call matter/energy. It also seems natural that a part of the vacuum becomes metrical to hold the embryonic universe and feed it, not unlike an ovary of a fruit.

Without a prior length scale, the universe presumably adapted the scale similarity with logarithmic modulations, which is typical for the turbulent flows [6]. So, the appropriate variable for the global evolution of the universe is $\eta(t)=\ln \left[a(t) / a_{0}\right]$, where $a(t)$ is the size of the universe (scale factor) as a 
function of time and subscript 0 indicates the present epoch $(t=0)$. Corresponding second order differential equation for the continuous evolution can be conveniently written in the form:

$$
\ddot{\eta}=-\xi \text {, }
$$

where dots indicate differentiations with respect to time and $\xi$ has dimension of inverse time squire: $[\xi]=T^{-2}$. For a limited growth, the simplest assumption about $\xi$ is that it is a positive constant. The general solution of (1) has the Gaussian form:

$$
a(t)=a_{0} \exp \left[H_{0} t-\frac{1}{2} \xi t^{2}\right]
$$

where $H_{0}=\dot{\eta}(0)$ is the Hubble constant. With $H_{0}>0$, this solution describes continuous growth from $a(-\infty)=0$ to maximum $a_{m}=a_{0} \exp \left[\frac{H_{0}^{2}}{2 \xi}\right]$ at $t_{m}=H_{0} / \xi$. For $t>t_{m}, a(t)$ continuously decreases to $a(-\infty)=0$. The main parameter of this model is $\xi$, because $H_{0}$ can be eliminated by shifting time. It seems natural to connect $\xi$ with gravity, which restrains the growth. In Ref. 7 it was shown, that universe with spatial dimension 1 and 2 collapses to singularity in a finite time. "Only starting with the spatial dimension 3, universe get an opportunity to survive" [7]. In the (3+1)-dimensional space-time, the gravitational constant $G$ has dimension $[G]=M^{-1} L^{3} T^{-2}$. Assuming that $\xi$ is proportional to $G$, we get $[\xi / G]=M L^{-3}$, which is the dimension of the mass density, which we denote $\rho_{0}$. So, we can write: $\xi=\theta G \rho_{0}$, where $\theta$ is nondimensional. If $\theta$ is constant, then $\rho_{0}$ is also constant and is important physical parameter, which determines the global dynamics of the universe. In the next section we will derive the described above model from the quantum modification of general relativity (Qmoger) and, based on the Qmoger equations, calculate the value $\theta=4 \pi$. It turns out, that such simple model, without any fitting, is in good quantitative agreement with cosmic data, including the acceleration [see below, particularly, Fig.1 and equation (11)].

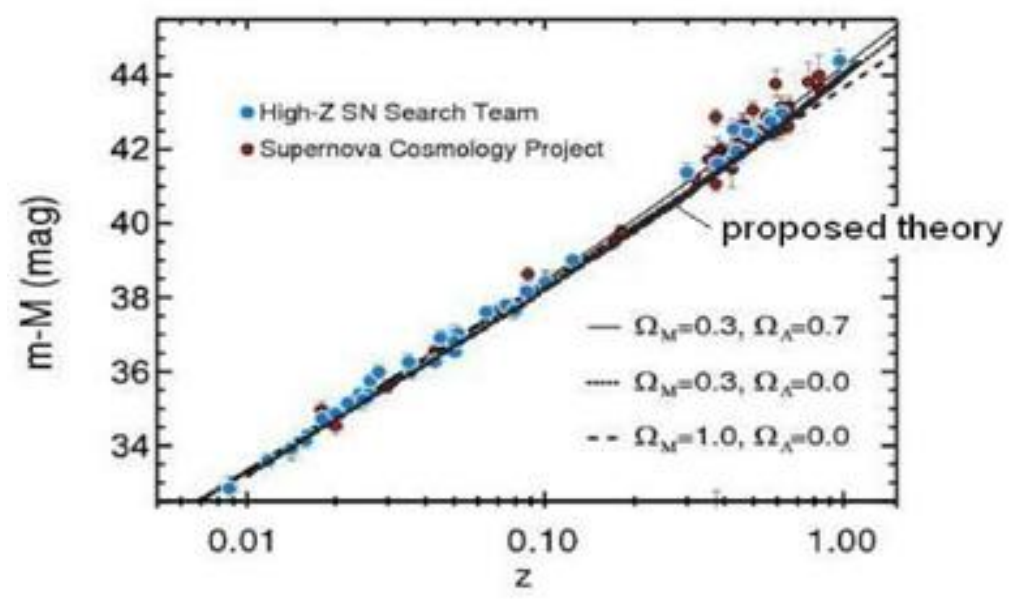

Figure1. Comparison of exact analytical solution $(2,10)$ with results of two observational projects and with some parametric models (details in Ref. 10,14). Here $z=\frac{a_{0}}{a}-1$ is the redshift, $m-M$ is the distance module as function of $z, m$ and $M$ are apparent and absolute magnitudes of the source correspondingly. The observations are model-independent

At this stage, we can conclude that gravity is the primary force. The vacuum can, potentially, create and absorb matter with various properties. Gravity serves the purpose of formation a finite compact object the universe. This requirement of the indicated above instability of the vacuum creates a natural selection of matter. Only gravitating matter survives this selection.

\section{QUANTUM MODIFICATION OF GENERAL RELATIVITY (QMOGER)}

Qmoger equations, introduced in Ref. 3 and discussed in more detail in Ref. 4 and 5, have the form:

$$
\begin{gathered}
R_{i}^{k}-\frac{1}{2} \delta_{i}^{k} \mathrm{R}=8 \pi G_{*} T_{i}^{k}+\lambda_{N} \delta_{i}^{k}, T_{i}^{k}=w u_{i} u^{k}-\delta_{i}^{k} p, w=\varepsilon+p \\
\lambda_{N}=\lambda_{0}+\beta \frac{d \sigma}{d s}+\gamma \sigma^{2}, \sigma=\frac{\partial u^{k}}{\partial x^{k}}+\frac{1}{2 g} \frac{d g}{d s}, \frac{d}{d s}=u^{k} \frac{\partial}{\partial x^{k}}
\end{gathered}
$$


Here $R_{i}^{k}$ is the curvature tensor, $p, \varepsilon=\rho c^{2}$ and $w$ are pressure, energy density and enthalpy density, respectively, $\rho$ is the mass density, $G_{*}=G c^{-4}\left(c\right.$ - speed of light), $u^{k}$-components of velocity (summation over repeated indexes is assumed from 0 to $3, x^{0}=c t$ ), $\lambda_{0}$ is the cosmological constant (which we will put zero), $\sigma$ is the covariant divergency, $\beta$ and $\gamma$ are nondimensional parameters (with particular choice $\beta=2 \gamma=2 / 3$, see below) and $g$ is the determinant of the metric tensor. With $\beta=\gamma=0$ we recover the classical equations of GR. Let us note that curvature terms in the left-hand side of (3) and additional terms $d \sigma / d s$ and $\sigma^{2}$ all contain second order (or square of first order) derivatives of metric tensor, which make these terms compatible. The importance of $\sigma$ also follows from the fact that it is the only dynamic characteristic of media, which enters into the balance of the proper number density of particles $n: \frac{d n}{d s}+\sigma n=q$, where $q$ is the rate of particle production (or absorption) by the vacuum. So, if $n$ is constant (see the exact analytical solution (10) below) or changing slowly, then the $\sigma$-efect is, certainly, very important in quantum cosmology. The $\sigma$-terms were introduced [3] with such physical argumentation on base of previous works [7-9]. Later, in the case $\beta=2 \gamma$, equations $(3,4)$ were derived from the variational principle by simply replacing the cosmological constant $\lambda_{0}$ (in the Lagrangian) by $\lambda=\lambda_{0}+\sigma^{2}[10]$. Indeed, the variation of $\int d^{4} x(-g)^{1 / 2} \sigma^{2}$ with respect to the metric tensor produces the two $\sigma$-terms in $(3,4)[10]$. But, the system is not Hamiltonian, the vacuum is feeding the universe, so, the standard approach is not appropriate ${ }^{1)}$. Parameters $(\beta, \gamma)$ generally, depend on the equation of state (see next section).

Some exact analytical solutions of equations $(3,4)$ where obtained in Ref. 3 . On the basis of these solutions, it was concluded that the effect of space time stretching $(\sigma)$ explains the accelerated expansion of the universe and for negative $\sigma$ (collapse) the same effect can prevent formation of singularity. Equations $(3,4)$ reproduce Newtonian gravitation in the nonrelativistic asymptotic, but gravitational waves can propagate with speed, which is not necessary equal to speed of light [10].This give us a hint that gravitons may have finite mass (below).

The natural next step was quantitative comparison with cosmological data and choice of parameters $\beta$ and $\gamma$. Let us consider equations for the scale factor $a(t)$ in homogeneous isotropic universe, derived from $(3,4)$ by standard procedure [Eq. $(8,9)$ in Ref. 3, or Eq. $(3,4)$ in Ref. 5]:

$$
\begin{aligned}
& \frac{(2-3 \beta) \ddot{a}}{a}+(1+3 \beta-9 \gamma)\left(\frac{\dot{a}}{a}\right)^{2}+\frac{k c^{2}}{a^{2}}-\lambda_{0} c^{2}=-8 \pi G p / c^{2} \\
& -\frac{\beta \ddot{a}}{a}+(1+\beta-3 \gamma)\left(\frac{\dot{a}}{a}\right)^{2}+\frac{k c^{2}}{a^{2}}-\frac{\lambda_{0} c^{2}}{3}=\frac{8 \pi}{3} G \rho
\end{aligned}
$$

Here the discrete curvature parameter $k=0,+1,-1$ corresponds to flat, closed and open universe, respectively.

With indicated in Ref. 3 unique choice $\beta=2 \gamma=2 / 3$, these equations take simple form:

$$
\begin{gathered}
\frac{k}{a^{2}}=\lambda_{0}-8 \pi G_{*} p \\
\ddot{\eta}=\frac{3 k c^{2}}{2 a^{2}}-\frac{\lambda_{0} c^{2}}{2}-4 \pi G \rho
\end{gathered}
$$

Where $\eta=\ln \left(a / a_{0}\right)$. From (7) with $\lambda_{0}=0$, we see that sign of curvature is opposite to sign of pressure. From observations we know that global curvature is close to zero. So, the dust approximation $(p=0)$ is natural for this theory with $\lambda_{0}=0$ and $\beta=2 \gamma=2 / 3$. In the dust approximation with $\lambda_{0}=0, k=0$, two special cases for system $(5,6)$ have been indicated [3]: 1) for $\beta=2 / 3$ and $\gamma \neq 1 / 3$ stationary solution exist; 2 ) for $\beta=2 \gamma$ the global energy is conserved, except for $\beta=2 \gamma=2 / 3$. The choice $\beta=2 \gamma=2 / 3$ is exceptional and in the dust approximation with $\lambda_{0}=0, k=0$, equation (7) is identity and (8) reduces to:

$$
\ddot{\eta}=-4 \pi G \rho
$$

In order to solve this equation, we need an assumption about $\rho$. For a continuous creation of matter by the vacuum, the simplest situation is with constant mass density $\rho=\rho_{0}$. In Ref. 5 and in the next section some more general situations are considered. With constant mass density, from (9) we have exact analytical Gaussian solution:

$$
a(t)=a_{0} \exp \left[H_{0} t-2 \pi G \rho_{0} t^{2}\right]
$$


So, in this case, from Qmoger we got the same solution as in the model (2), but now with fixed constant $\xi=4 \pi G \rho_{0}$. In the analogous solution, obtained in Ref.10, instead of $\varepsilon_{0}$ was $\rho_{0}+\left(\lambda_{0} c^{2}\right) /(8 \pi G)$, for generality.

We use value $\rho_{0}=2.6 \cdot 10^{-30} \mathrm{gcm}^{-3}$, which, according to WMAP [12], includes ordinary and dark matter. We do not include the dark energy, which does not exist in Qmoger (see below). For the characteristic time scale we have $\left(G \rho_{0}\right)^{-1 / 2}=T_{*} \approx 76$ billion years $(b y)$. Corresponding length scale $L_{*}=c T_{*} \approx 76$ billion light years (bly) is comparable with the current size of the visible universe $a_{0} \approx 46.5$ bly. We use recently measured [13] value $H_{0}=70.0(\mathrm{~km} / \mathrm{s}) / M p c \approx 2.27 \cdot 10^{-18} \mathrm{~S}^{-1}$. Remarkably, $T_{*} H_{0} \approx 5.27$. The temporal scale $H_{0}^{-1}$ and the eternal scale $T_{*}$ are of the same order because currently $a(t)$ is relatively close to its maximum $a_{m} \approx 3.24 a_{0}$ at time $t_{m}=\left(T_{*}^{2} H_{0}\right) /(4 \pi) \approx 32.9$ by. It was shown [10], that solution (10) is globally stable during expansion $\left(-\infty<t<t_{m}\right)$. But, after that it becomes unstable and additional investigation is needed for evolution of the universe at $t>t_{\max }$

From (10) we get current acceleration parameter:

$$
\frac{\ddot{a}_{0} a_{0}}{\left(\dot{a}_{0}\right)^{2}}=1-\frac{4 \pi G \rho_{0}}{H_{0}^{2}}=0.58
$$

So, we do not need the dark energy in order to explain the acceleration. According to (10), with indicated above values of $H_{0}$ and $\rho_{0}$, the maximum of velocity $\dot{a}=0.093 c$ will be at $t \approx 11.7 b y$. Note, that measurements of $H_{0}$, generally, give different results for different galaxies and the background radiation is anisotropic. According to Qmoger, it seems natural. Formation of galaxies and corresponding radiation are results of gravitational instability of the background quantum condensate (below). So, galaxies do not have to be synchronized during expansion of the universe. The same is true for radiation. The BB theory has difficulties to explain these cosmic observations. The BB theory also has difficulty to explain some old structures in the universe, for example, recently discovered an 800-million-solar-mass black hole in a significantly neutral universe at redshift $z=a_{0} / a-1=7.5[14]$.

Solution (10) does not have any fitting parameters and is in good quantitative agreement with cosmic data $[10,15,4]$, see also Fig. 1 . This solution eliminates not only singularity of Big Bang, but also other major controversies - critical density of the universe (which is difficult to explain physically), dark energy (ridiculously small cosmological constant) and inflation (which does not sit well with Big Bang). Remarkably, solution (10) does not depend on the speed of light $c$. That is because, according to Qmoger, there was not much light in the beginning. Photons and other "ordinary" matter were selected and actively produced much later during formation of galaxies (see below). Even at the later stages, the contribution of light into the total energy of the universe is small.

\section{ISENTHALPIC UNIVERSE}

To consider the effect of radiation on the global dynamics, we rewrite equations $(5,6)$ in the form:

$$
\begin{gathered}
\ddot{\eta}=-4 \pi G w c^{-2}+\mathrm{kc}^{2} / a^{2}, \\
8 \pi G p=3(3 \gamma-1) \dot{\eta}^{2}+4 \pi(2-3 \beta) G \mathrm{w}-\frac{3(1-\beta) k c^{4}}{a^{2}}+\lambda_{0} \mathrm{c}^{4} .
\end{gathered}
$$

Note, that equation (12) does not have parameters $\lambda_{0}, \beta, \gamma$ and is the same for the classical GR. In order to solve the system $(12,13)$, we need some form of the equation of state. The popular approximation $p=\varkappa \varepsilon$ (with constant $\mathcal{~}$ ) was considered in Ref. 5. Equation (12) suggests a different possibility. A novel approximation is to assume that the enthalpy density is constant: $w=\rho c^{2}+p=w_{0}$. This will allow the pressure to grow and a part of the energy to radiate, for example, during formation and interaction of stars. With that approximation, we can solve equation (12) for any curvature parameter $k$ and get pressure from (13). Particularly, for $k=0$, equation (12) gives:

$$
\dot{\eta}=H^{0}-\frac{4 \pi G w^{0} t}{c^{2}}, \quad a(t)=a_{0} \exp \left[H_{0} t-\frac{2 \pi G w_{0} t^{2}}{c^{2}}\right]
$$

This solution differs from (10) by substitution $w_{0} c^{-2}$ instead of $\rho_{0}$. The difference is small, because the averaged pressure in the universe is small in comparison with the energy density. For solution of Einstein equation $(\beta=\gamma=0)$ with $k=0$, from $(13,14)$ we get:

$$
8 \pi G \varepsilon=3\left(H_{0}-\frac{4 \pi G w_{0} t}{c^{2}}\right)^{2}-\lambda_{0} c^{4}, p=w_{0}-\varepsilon .
$$

From (15), we see that $\varepsilon \rightarrow \infty$ and $p \rightarrow-\infty$ with $t \rightarrow \pm \infty$, which is unphysical. 
In Qmoger we can avoid such contradiction with reality. During formation and interaction of stars, parameters $\beta$ and $\gamma$ are, generally, no longer constant. For isenthalpic processes $\left(w=w_{0}\right)$ we can assume $(\beta, \gamma)$ to be functions of $p / w_{0}$. A simple model for deviation from used above values $\beta=2 / 3, \gamma=1 / 3$, has the form:

$$
\beta=\frac{2}{3}\left[1-b\left(\frac{p}{w_{0}}\right)^{2}\right], \quad \gamma=\frac{1}{3}\left[1+x\left(\frac{p}{w_{0}}\right)^{2}\right]
$$

where $b$ and $\chi$ are positive constants. Substitution of (16) into (13), with $\lambda_{0}=0$ and $k=0$, gives nontrivial solution:

$$
\frac{p}{w}=\frac{8 \pi G w_{0}}{8 \pi G w_{0} b+x\left(H_{0} c-4 \pi G w_{0} c^{-1} t\right)^{2}} \quad \varepsilon=w_{0}-p
$$

According to (17), the averaged pressure increases from 0 at $t=-\infty$, riches maximum $p_{m}=w_{0} / b$ at $t_{w}=H_{0} c^{2} /\left(4 G w_{0}\right)$ and decreases to 0 at $t=\infty$. Averaged energy density starts with $\varepsilon=w_{0}$ at $t=-\infty$, decreases to minimum $w_{0}(1-1 / b)$ at $t_{w}$ and then return to the original value $w_{0}$. Such behavior of averaged pressure and energy seems physically reasonable. Part of the energy, radiated during formation and interaction of stars, absorbs in contraction. Positivity of energy $(\varepsilon>0)$ gives restriction on parameter $b>1$.

Choice of isenthalpic process is dictated by the form of Einstein and Qmoger equations and leads to simple Gaussian solution (14). We can speculate that such processes are wide spread in Nature and wait to be discovered.

Let us stress, that solution of Einstein equations (with $\beta=\gamma=0$ ) and solution of Qmoger equations give the same result (14), corresponding to cosmic data with $w$ close to $\varepsilon$ (see Figure 1). But asymptotics $\varepsilon \rightarrow \infty$ and $p \rightarrow-\infty$ with $t \rightarrow \pm \infty$ in solution of Einstein equation (15) looks unphysical.

Formulas (16) can be used also for local calculation of galaxies formation with Qmoger equations. More details can be obtained by inclusion electromagnetic terms into Qmoger equations. Isenthalpic processes of dipolar gravitons (see below), can produce radiation and magnetic field not only in stars (such as our Sun), but also in a planet core. Excessive radiation of Jupiter and Saturn [16] could be connected with such phenomena. More details can be obtained by introducing into the basic equations $(3,4)$ additional fields (Qmoger+), particularly, the electromagnetic field.

\section{ENTANGLED UNIVERSE: QUANTUM CONDENSATE OF GRAVITONS - DARK MATTER, CRITICAL SIZE OF CONDENSATE AND QUANTUM GRAVITATIONAL WAVES}

In seems natural, that the quantum interaction was selected in order to keep the early universe as a compact quantum condensate, which will resist the gravitational instability. The quantum uncertainty condition with indicated above time scale $T_{*}$ corresponds to energy $E_{0}=\hbar / T_{*} \simeq 4: 17 \cdot 10^{-46} \mathrm{erg} \simeq 2.61 \cdot 10^{-34} \mathrm{eV}$, where $\hbar$ is the Planck constant. That is the unique energy, determined by parameters $G, \rho_{o}$ and $\hbar$. Corresponding mass of a particle is $m_{0}=\hbar /\left(c^{2} T_{*}\right) \simeq 4.6 \cdot 10^{-67} \mathrm{gram}$. This particle we call graviton, because only gravity field was used in the theory. In this paper we will not compare such definition of graviton with many definitions and use of gravitons in the literature [17]. Let us only stress, that Qmoger theory is not Hamiltonian (see section 3). Gravitons in this theory are not virtual, but real particles with small, but finite mass $m_{0}$ [compare with electron mass $m_{e} \simeq 9 \cdot 10^{-28} \mathrm{gram}$ ] and, possibly, with some electromagnetic properties (see section 7).

Taking into account, that the dark matter dominates the mass content of the universe, the concentration of particles $n$ and characteristic scale $l$ (averaged distance between particles) can be estimated as:

$$
n=\rho_{0} / m_{0} \approx 5 \cdot 10^{36}, l=n^{-1 / 3} \approx 2.7 \cdot 10^{-13} \mathrm{~cm} .
$$

Such particles will form the quantum condensate even for high temperature $[4,5]$. Indeed, the thermal de Brogle wavelength for the temperature of the universe $T \approx 2.72 \mathrm{~K}$ is many orders bigger than l: $\hbar c /\left(k_{B} T l\right) \approx 3 \cdot 10^{11}\left(k_{B}\right.$-Boltzmann constant). This estimate is for massless particles. For non relativistic gravitons with indicated above mass $m_{0}$, the corresponding factor is even bigger: $\hbar\left(m_{0} k_{B} T\right)^{-1 / 2} l^{-1} \approx 7 \cdot 10^{13}$. So, gravitons are entangled in the quantum condensate.

To estimate time $t_{g}$, when such gravitons can be created, we use condition

$a\left(t_{g}\right)=l$. From (10) we have:

$$
t / T_{*}=\zeta \pm\left[\zeta^{2}-\frac{\eta}{2 \pi}\right]^{1 / 2}, \quad \zeta=\left(T_{*} H_{0}\right) /(4 \pi) \approx 0.43 .
$$


Here minus correspond to the past. For $\eta_{g}=\ln \left(l / a_{0}\right) \approx 91$, from (19) we got $t_{g} \approx-258$ by. For a while, the growing universe, supported by the quantum interaction, will resist the gravitational instability. The critical size of the quantum gravitating condensate $l_{c r}$, for arbitrary $\rho$, is uniquely determined by parameters $(G, \rho, \hbar)$. Namely:

$$
l_{c r}=\hbar^{1 / 5} G^{-1 / 10} \rho^{-3 / 10}
$$

As expected, $l_{c r}$ is increasing with increase of $\hbar$ and decreasing with increase of $G$ and $\rho$. Particularly, at $\rho=\rho_{0}$, we get $l_{c r, 0} \simeq 1.5 \cdot 10^{6} \mathrm{~cm}=15 \mathrm{~km}$. Corresponding time $\left(t_{c r}\right)$, when embryonic galaxies start to form, is determined by (19) with $\eta_{c r}=\ln \left(l_{c r, 0} / a_{0}\right) \approx-51$.7. The result is $t_{c r} \approx-187.5$ by. So, it took 70.5 by of graviton production to initiate formation of galaxies. Corresponding critical mass $m_{c r}=\rho_{0} l_{c r, 0}^{3}=$ $\hbar^{3 / 5} G^{-3 / 10} \rho_{0}^{1 / 10} \approx 8.7 \cdot 10^{-12}$ gram. Important nondimensional parameter of the theory is the critical number of gravitons $N_{c r}=\frac{m_{c r}}{m_{0}}=\hbar^{-2 / 5} G^{-4 / 5} \rho_{0}^{-2 / 5} c^{2}=\left[\frac{c}{u_{\hbar, 0}}\right]^{2} \approx 1.9 \cdot 10^{55}$. Here $u_{\hbar, 0}=\left(\hbar G^{2} \rho_{0}\right)^{1 / 5} \approx 6.5$. $10^{-15} \mathrm{cms}^{-1}$. This is a particular case of speed $u_{\hbar}=\left(\hbar G^{2} \rho\right)^{1 / 5}$ of quantum gravitational waves in the condensate, which is uniquely determined by parameters $(G, \rho, \hbar)$. The obtained small value of $u_{\hbar, 0}$ corresponds to small averaged mass density $\rho_{0}$. During formation of galaxies and interactions of stars, local $\rho$, potentially, can approach the Planck density $\rho_{P}=c^{5} /\left(\hbar G^{2}\right)$ and local $u_{\hbar}$ can be comparable with $c$. Formula (20) can be applied to the blobs of dark matter in the halo of galaxies. In the isenthalpic approximation (above), $\rho$ is replaced by $w c^{-2}$, particularly, in the expressions for $l_{c r}$ and $u_{\hbar}$

\section{CREATION OF NEUTRINOS FROM THE BACKGROUND GRAVITONS}

Due to the collapsing mechanism, described in Ref. 7, some hot spots were developing during formation of galaxies. So, there is a necessity to get rid of an excessive energy. Apparently, photons were selected out of this necessity.The next step in getting rid of excessive energy is selection of fermionic massive particles, which obey the Pauli exclusive principle and will run away from hot spots. Apparently, creation of new particles does not depend directly from gravity. We see it on example of photons, which are characterized only by parameters $c$ and $\hbar$. From these two parameters, which have dimensions $[c]=L T$ ${ }^{1}$ and $[\hbar]=M L^{2} T^{-1}$, we can't construct a mass. That is why, the Standard Model can't predict absolute value of mass for observable particles. If $G$ is taking into account, parameters $(c, \hbar, G)$ give uniquely the Planck mass $m_{P}=\left(\frac{\hbar c}{G}\right)^{1 / 2} \approx 2.18 \cdot 10^{-5}$ gram, which is many orders heavier than observable particles (for example, mass of electron $m_{e} \approx 9.11 \cdot 10^{-28} \mathrm{gram}$ ).

In Qmoger theory, from parameters $\left(c, \hbar, \rho_{0}\right)$ we get unique mass:

$$
m_{*}=\rho_{0}^{1 / 4}(\hbar / c)^{3 / 4} \approx 3.13 \cdot 10^{-36} \mathrm{gram} \approx 1.76 .10^{-3} \mathrm{eV} / \mathrm{c}^{2}
$$

From the same parameters, we get unique length scale:

$$
l_{*}=\frac{\hbar}{c m_{*}}=\left[\frac{m_{*}}{\rho_{0}}\right]^{1 / 3}=\left[\frac{\hbar}{c \rho_{0}}\right\}^{1 / 4} \approx 10^{-2} \mathrm{~cm}
$$

The obtained scale $l_{*}$ corresponds to the Compton wavelength of a particle with mass of background matter occupying volume of size $l_{*}$. This indicates a mechanism of formation new particles from the background matter. We associate the obtained mass (21) with the first and the lightest massive fermionic particle created from the background condensate. The best candidate for that role from the observable particles is neutrino. Indeed, $m_{*}$ satisfies the experimental upper limit for neutrinos about $4 \cdot 10^{-2} \mathrm{eV} / \mathrm{c}^{2}$ [17]. Taking into account that $m_{*}$ is obtained from dimensional analysis, a moderate numerical factor is expected. The time scale:

$$
t_{*}=\left[\frac{\hbar}{c^{5} \rho_{0}}\right]^{1 / 4} \approx 3.3 \cdot 10^{-13} s
$$

can be associated with formation and acceleration $\left(\mathrm{c} / t_{*} \approx 8.46 \cdot 10^{-22} \mathrm{Cms}^{-2}\right.$ ) of neutrino, as well as to the neutrino oscillations (transformation between three flavours) [18]. The physics of these oscillations can be related to interaction of neutrino with the background condensate of ultralight gravitons. The average number of gravitons interacting with such neutrino can be estimated by $N_{*}=m_{*} / m_{0} \approx 10^{30}$. Below we will estimate the electric dipole moments (EDM) of both gravitons and neutrinos, which can be a significant 
factor of interactions. During its flight, neutrino can create some disturbances in the background condensate and temporary carry along coherent groups of gravitons, perhaps, in a form of vortex ring. This will influence the effective mass and the flavor of neutrino [17]. This is an example of interface between dark and ordinary matter (Idom), introduced in context of explanation of the phenomena of subjective experiences (qualia) [19] (below). Note, that in frames of the Standard Model, the stability of all three neutrinos $\left(v_{e}, v_{\mu}, v_{\tau}\right)$ was a longtime puzzle. In Qmoger, stability of the first-generation particles (neutrinos), produced by the background condensate, is natural.

\section{Electric Dipole Moment of Graviton And Neutrino.}

The baryonic asymmetry of the universe (prevalence of matter over antimatter) can be explained if gravitons have nonzero electric dipole moment (EDM). Indeed, EDM of primary particles can break the reflection symmetry and give advantage to matter over antimatter. It will also help to explain synthesis of some particles from the dipolar quantum condensate. Additionally, EDM of gravitons helps to explain qualia [19] and brightens the dark sector of matter (see below).

Technically, it is simpler to estimate EDM of neutrino, because, as explained above, properties of neutrino do not depend directly of $G$. From parameters (c, $\hbar, \rho_{0}$ ) we get unique expression for EDM of neutrino or similar first-generation particle, produced by the background condensate:

$$
d_{*}=\left[\frac{\hbar^{3} c}{\rho_{0}}\right]^{1 / 2} \approx 5.8 \cdot 10^{-11} \operatorname{gram}^{\frac{1}{2}} \mathrm{~cm}^{\frac{5}{2}} \mathrm{~s}^{-1} \approx 0.12 \mathrm{ecm} \text {. }
$$

For gravitons we have additional parameter $G$. The general expression for EDM of graviton, from dimensional argument, can be written in the form: $d_{0}=d_{*} F\left(\frac{m_{0}}{m_{*}}\right)$ with arbitrary function $F$. If we assume that EDM of graviton is proportional to its mass, than $d_{0} \sim 1.2 \cdot 10{ }^{-29} \mathrm{ecm}$. In Ref. 4, 5 it was suggested that $d_{0} \sim m_{0}^{\frac{1}{2}} l_{P}^{\frac{3}{2}} c \sim 2 \cdot 10^{-72} \operatorname{gram}^{\frac{1}{2}} \mathrm{~cm}^{\frac{5}{2}} S^{-1}$. Here $l_{P}=\left\{\frac{\hbar G}{c^{3}}\right]^{1 / 2} \approx 1.6 \cdot 10^{-37} \mathrm{~cm}$ is the Planck length. In any case, $d_{0}$ is very small and conventional telescopes can't see gravitons (dark matter). It also very difficult to detect gravitons in the supercollider or in any high energy machine. But, in section 9 we consider a novel approach to study gravitons.

\section{VACUMO}

The solution (10) of the partial differential equations $(3,4)$ is valid under condition $a(t) \geq l_{P}$, which corresponds to sufficiently smooth space-time. In frames of Qmoger, the time $t_{1}$ of the beginning of the universe is naturally determined by equation $a\left(t_{1}\right)=l_{P}$. From $(10)$, we get $t_{1} \approx-327$ by $[20,4,5]$. So, there was $t_{g}-t_{1} \approx 68 b y$ of feeding the infant universe before it was able to produce gravitons. The time $t_{1}$ corresponds to the mass of the universe $M_{1}=\rho_{0} l_{P}^{3} \approx 10^{-128} \mathrm{gram}$ (recall, that the mass of graviton $m_{0} \approx 4.6 \cdot 10^{-67} \mathrm{gram}$ ). That results suggest existence of particles or quasi particles with energy smaller than $E_{0} \approx 2.6110^{-34} \mathrm{eV}$. If such particle has the rest mass, it is much smaller than $m_{0}$. Any such particle we call vacumo. Apparently, the universe is feeding by vacumos, which than transform into gravitons. Vacumos also can play a role of mediators between gravitons and ordinary matter, particularly, in formation of neutrinos (see above).

In Ref. 10 is was argued, that dark matter particle (which we now call gravitons, see above) consists of a pair of massive photons. They do not have to be ordinary photons, but photon-like particles. That is consistent with EDM of gravitons (see above).

\section{QUALIA}

In our subjective experiences (qualia) we can actually see and feel the collective effects of the background gravitons [19]. The acting potentials of neurons [21], apparently, create gaps and coherent patterns in the condensate of gravitons. Remarkably, these interfaces between dark and ordinary matter (Idom) in cases of neutrino and qualia are similar. Note, that length scale (22) is of the same order as the size of the neuron cluster, which can produce sufficiently rich qualia. The necessary for qualia huge number of degrees of freedom is supplied by the indicated in above number of gravitons $N_{*} \approx 10^{30}$. It will not be surprising if oscillating neutrinos, which interact with gravitons (see above), play a role in qualia along with the acting potentials of neurons. At the same time, artificial sources of neutrino in a laboratory setting, potentially, can be used for healing and stimulation of the brain. By manipulating with acting potentials and quantifying qualia responses, we can open a new window into the dark sector of matter. 


\section{CONCLUSIONS}

This paper presents a beginning of a new type of theory, in which the laws of nature are evolving along with the evolution of the universe. The laws of nature seem to be approximate in connection with the feeding of the universe by the vacuum and because of the indicated above instabilities (see also below). Particularly, the conservation of energy emerged when there was already enough energy, so, that additional feeding has small effect on the local balance of energy. The universe is an open (nonHamiltonian) system. The evolution of the universe was started with selection of the (3+1)-dimensional space-time and gravity, as a stable situation for the beginning. Then came the quantum condensate of entangled gravitons for an additional stabilization. When this condensate becomes gravitationally unstable, the production of ordinary matter (photons, neutrino and more heavy particles) was selected as a relieve from excessive energy in hot spots. The phenomena of quantum entanglement of ordinary matter is, apparently, inherited from the background condensate. From the human point of view, the highest present achievement of the developing universe, of course, is qualia, which was produced by the interface between gravitons and ordinary matter.

In future, we can combine the Standard model and the Quantum Field Theory with Qmoger. This will definitely open new directions of research in physics and biophysics.

1)Note, that Newton and Einstein did not use the Lagrangian, the Hamiltonian and the variational principle. Unfortunately, these days the physical and common sense are often replaced by the variational principle. We can blame textbooks, which are convenient to base on the variational principle. In my opinion, it can lead theoretical physics astray. The non-Hamiltonian Qmoger theory with its seeping gravitons, apart from correcting cosmology, could also help to correct some of deficiencies in the Quantum Field Theory, particularly, the inequivalent representations [11]. Indeed, the active background can eliminate unstable representations of reality.

\section{REFERENCES}

1. https://en.wikipedia.org/wiki/Big_Bang

2. P. J. Steinhardt, The inflation debate: Is the theory at heart of modern cosmology deeply flawed?, Scientific American, April; pp. 18-25 (2011).

3. E. A. Novikov, Vacuum response to cosmic stretching: accelerated universe and prevention of singularity arXiv:nlin/06080050.

4. E. A. Novikov, Ultralight gravitons with tiny electric dipole moment are seeping from the vacuum, Modern Physics Letters A, 31, No. 15, 1650092 (5 pages) (2016).

5. E. A. Novikov, Quantum modification of general relativity, Electr. J. Theoretical Physics, 13, No. 35, 7990, (2016).

6. E. A. Novikov, Mathematical Model for the Intermittency of Turbulent ow, Dokl. Akad.Nauk SSSR 1966,168, 1279-1282 [Sov. Phys. Dokl. 1966,11, 497-500]; Scale Similarity for Random Fields, Dokl. Akad. Nauk SSSR 1969, 184, 1072-1075 [Sov. Phys. Dokl. 1969,14(2), 104-107]; Intermittency and Scale Similarity in the Structure of a Turbulent Flow, Prikl. Mat. Mekh. 1971, 35, 266-275 [Appl. Math. Mech. 1971, 35, 231-240]; The Effects of Intermittency on Statistical Characteristics of Turbulence and Scale Similarity of Breakdown Coefficients, Phys. Fluids A 1990, 2(5), 814-820; Infinitely Divisible Distribution in Turbulence, Phys. Rev. E 1994, 50(5), R3303-R3309.

7. E. A. Novikov, Nonlinear evolution of disturbances in (1+1)-dimensional universe, Zh. Exper. Teor. Fiz. 57, 938 (1969) [Sov. Phys. JETP. 30 (3), 512 (1970)]; arXiv:1001,3709 [physics.gen-ph].

8. E. A. Novikov, Dynamics of distributed sources, Physics of Fluids 15, L65 (2003).

9. E. A. Novikov, Distributed sources, accelerated universe and quantum entanglement, arXiv:nonlin.PS/0511040.

10. S. G. Chefranov\& E. A. Novikov, Hydrodynamical vacuum sources of dark matter self-generation without Big Bang, J. Exper. Theor. Phys., 111(5),731-743 (2010) [Zhur. Eksper. Theor. Fiz.,138(5), 830-843 (2010)]; arXiv:1012.0241v1 [gr-qc].

11. https://plato.stanford.edu/entries/quantum- eld-theory/\#DefStaForQFT

12. https://map.gsfc.nasa.gov/

13. The LIGO Scientific Collaboration and The Virgo Collaboration; The 1M2H Collaboration; The Dark Energy Camera GW-EM Collaboration and the DES Collaboration; The DLT40 Collaboration; The Las 
Cumbres Observatory Collaboration; The VINROUGE Collaboration; The MASTER Collaboration (2017-10-16). "A gravitational-wave standard siren measurement of the Hubble constant". Nature. advance online publication. doi:10.1038/nature24471. ISSN 1476-4687.

14. E. Baæados et al, An 800-million-solar-mass black hole in a significantly neutral universe at redshift 7.5, Nature 25180, 6 December 2017; arXiv:1712.01860.

15. E. A. Novikov \& S Chefranov, A quiet cosmology and halo around the visible universe, J. of Cosmology $16,6884(2011)$

16. https://solarsystem.nasa.gov

17. https://en.wikipedia.org/wiki/Graviton.

El-Nabulsi, Rami Ahmad, Gravitons in Fractional Action Cosmology, Int. J. Theor. Phys. 51 (2012) 3978.

R. Casadio, A. Giugno, A. Giusti, Matter and gravitons in the gravitational collapse, Phys. Lett. B763 (2016) 337.

C. de Pham, J. T. Deaskins, A. J. Tolley and S-Y. Zhot, Graviton mass bounds, Rev. Mod. Phys. 89 (2017), 025004.

A.F. Zakharov, P. Jovanovicc, D. Borka and V. B. Jovanovic, Graviton mass trajectories of bright stars at the Galactic Center, J. Phys.: Conf. Ser.017) 01081.

18. https://en.wikipedia.org/wiki/Neutrino

19. E. A. Novikov, Gravicommunication, subjectivity and quantum entanglement, NeuroQuantology, v. 14, issue 4, 677-682 (2016).

20. E. A. Novikov. Age of the universe and more, J. of Cosmology v.25, 13442-13452.

21. https://en.wikipedia.org/wiki/Action-potential.

Citation: Evgeny A. Novikov. "Emergence of the Laws of Nature in the Developing Entangled Universe", American Research Journal of Physics, 4(1): 1-9.

Copyright (C) 2018 Evgeny A. Novikov, This is an open access article distributed under the Creative Commons Attribution License, which permits unrestricted use, distribution, and reproduction in any medium, provided the original work is properly cited. 